\title{
Bases para una Estrategia Nacional de Clasificación en Deporte Paralímpico en España
}

\author{
Basis for a National Classification Strategy in \\ Paralympic Sport in Spain
}

\section{Palabras Clave}

Deporte paralímpico, para-deporte, clasificación funcional, discapacidad, Juegos Paralímpicos.

\section{Keywords}

Paralympic sport, para-sport, functional classification, disability, Paralympic Games.

\section{Introducción}

La clasificación de deportistas con alguna discapacidad para la competición ha sido un tema de gran controversia en para-deporte ${ }^{1}$ (DePauw y Gavron, 2005; Paciorek, 20I I; Brittain, 20I0). El primer sistema de clasificación internacional reconocido para la salud y la función de una persona fue la Clasificación Internacional de Deficiencias, Discapacidades y Minusvalías (OMS, I980), evolucionada actualmente al Sistema Internacional de Clasificación de Funcionalidad, Discapacidad y Salud -CIF- (International Classification of Functioning, Disability and Health-ICF) (OMS, 200I). La CIF ha permitido transformar una clasificación basada en el déficit a una clasificación basada en competencias de componentes de salud. Así, el término funcionalidad (functioning) se refiere a todas las funciones corporales, actividades y de participación, mientras que el de discapacidad (disability) sería su 'antítesis' en cuanto a deficiencias, limitación en las actividades y restricciones en la participación. Ello supone un cambio relevante sobre cómo se entiende actualmente la

I. Cualquier deporte adaptado para personas con discapacidad, ya sea paralímpico o no.

\section{Raúl Reina Vaíllo \\ <rreina@goumh.es> \\ Universidad Miguel Hernández}

\section{Nuria Vilanova-Périz}

<nuriavilanovaperiz@gmail.com>

Universidad Miguel Hernández

Para citar:

Reina, R. y Vilanova-Périz, N. (20I7):

"Bases para una Estrategia Nacional de Clasificación en Deporte Paralímpico en España”. Revista Española de Discapacidad, 5 (I): I95-2I6.

Doi: <https://doi.org/I0.5 569/23405IO4.05.OI.II > 
discapacidad, cambiando el foco de atención al estado de salud y capacidades del usuario (Reina, 20I4a). Además, la CIF provee el uso de un lenguaje estandarizado y un marco teórico para la descripción de diferentes estados relacionados con la salud, pero su aplicación en la actualidad va más allá del ámbito sanitario.

En para-deporte, "la propuesta de la clasificación debe ser proveer a los deportistas con discapacidad un punto de partida equitativo para que la competición se desarrolle en igualdad de condiciones" (Tweedy, 2002: 222). Ligándolo con la CIF, ésta puede considerarse como la organización de las deficiencias específicas de estructura y función en clases, basado en qué medida esas deficiencias (o impedimentos) hacen que el deportista experimente una limitación en la actividad en una disciplina en concreto (Tweedy, 2002). Entender cómo la discapacidad afecta al rendimiento pasa por identificar los determinantes de dicho rendimiento en cada para-deporte.

En los primeros años del Movimiento Paralímpico, la clasificación deportiva estaba basada en sistemas médicos, muy relacionados con la rehabilitación en el ámbito hospitalario. Estas clases dividían a los deportistas por tipos de deficiencia y servían para todos los deportes, pudiendo encontrar dentro de la misma clase diversos niveles de severidad en cuanto a su impacto en el rendimiento deportivo (Reina, 20I4b). Así, había sistemas para lesión medular $\left(\mathrm{ISMWF}^{2}\right)$, amputaciones y les autres (ISOD $\left.{ }^{3}\right)$, ceguera y deficiencia visual $\left(\mathrm{IBSA}^{4}\right)$, parálisis cerebral (CPISRAs), además de discapacidad auditiva $\left(\mathrm{ICSD}^{6}\right)$ y discapacidad intelectual (Special Olympics e INAS7) (Sanz y Reina, 20I 2). Algunos de estos sistemas o estructuras organizativas perduran en parte en la actualidad.

2. International Stoke Mandeville Wheelchair Sports Federation.

3. International Sports Federation for the Disabled.

4. International Blind Sports Federation.

5. Cerebral Palsy International Sports and Recreation Asso-

ciation.

6. International Commission of Sports for the Deaf

7. International Federation for Intellectual Disability Sport.
Entre el curso olímpico de Seúl i988 y Barcelona I992 se llegó a un acuerdo para que todos los deportes paralímpicos clasificaran a sus deportistas usando sistemas de clasificación funcionales. En 2003, el Comité Paralímpico Internacional (IPC) aprobó una Estrategia de Clasificación, que dio lugar al primer Código de Clasificación y Estándares Internacionales (IPC, 2007). Este Código describe que los sistemas de clasificación deben realizarse a través de investigaciones multidisciplinares, debiendo adecuarse a las habilidades y requerimientos de cada deporte. Tras un proceso de revisión de tres años (20I3-20I5), en el que han participado federaciones internacionales y comités paralímpicos nacionales, en noviembre de 20 I 5 se ha publicado el nuevo Código de Clasificación de Deportistas (IPC, 2015a), más centrado en el propio deportista, y al que se unen cinco estándares internacionales sobre deficiencias elegibles (IPC, 20I6a), evaluación de los deportistas (IPC, 20I6b), formación y acreditación de clasificadores (IPC, 20I6c), protestas y apelaciones (IPC, 20I6d), y protección de datos personales (IPC, 20I6e). Este nuevo Código de Clasificación de Deportistas y Estándares Internacionales tienen dos funciones clave:

- definir quién es elegible para competir en un determinado deporte paralímpico y, por consiguiente, tener la oportunidad de convertirse en deportista paralímpico; y

- agrupar a los deportistas en clases, con el objetivo de asegurar que el impacto de la deficiencia es minimizado y la excelencia deportiva es lo que determina el rendimiento o victoria de un deportista o equipo.

La Figura I ilustra la evolución de los sistemas de clasificación, desde el origen del deporte paralímpico en el ámbito hospitalario y sistemas de clasificación basados en criterios exclusivamente médicos, a la situación actual donde los sistemas deben ser específicos de cada para-deporte y basados en evidencias científicas (IPC, 20I5a). 


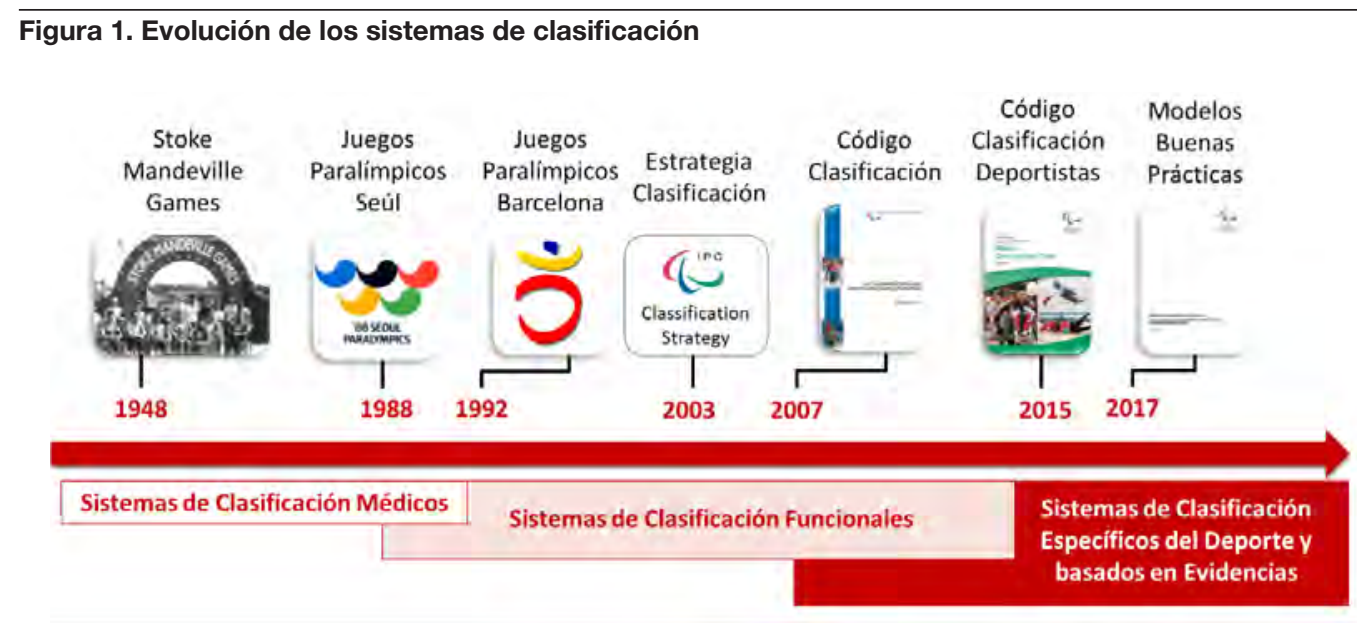

Fuente: adaptado de Hart (2017).

La propia evolución del deporte paralímpico ha conllevado la búsqueda e identificación de los factores que pueden condicionar el proceso y/o rendimiento de un deportista, por lo que Tweedy y Vanlandewijck (20I I) sintetizan dos postulados que deben sustentar cualquier proceso de clasificación:

- Nivel de función del deportista. Aunque la función se ve afectada por la deficiencia de la persona (e.g. la presencia de espasticidad en la cadera limitará la amplitud de zancada), existen otra serie de factores que influyen en la funcionalidad de la persona, tales como la edad, el nivel de entrenamiento o la motivación.

- Potencial de rendimiento o componente innato. Como en el resto de modalidades deportivas, existen una serie de condicionantes biológicos que pueden favorecer o contribuir al éxito. Así, por ejemplo, aquellos corredores con una mayor amplitud de zancada o con una mayor longitud de miembros inferiores podrían tener un mejor resultado en una carrera de velocidad.

El empeño en el desarrollo de sistemas de clasificación específicos de cada para-deporte se debe a las diferencias de rendimiento que pueden darse para una misma persona en diferentes deportes, atendiendo a las características que componen al deporte en cuestión y al impacto que la discapacidad puede tener sobre el mismo. Además, siguiendo los principios del movimiento olímpico sobre fair play, los sistemas de clasificación deben asegurar que el éxito de un deportista derive de la combinación de sus características antropométricas, fisiológicas y/o psicológicas y cómo es capaz de sacar el mejor resultado de éstas, y no porque éstos partan de una situación desventajosa de competición originada por el propio sistema de clasificación (Tweedy et al., 20I4).

En el contexto actual, en enero de 20I7, IPC aprobó dos documentos de gran relevancia para con los objetivos de este trabajo: I) un modelo de buenas prácticas para la estandarización de las reglas de clasificación entre las diferentes federaciones internacionales (IPC, 20I7a), y 2) otro para la aplicación de políticas y estándares de clasificación a nivel nacional (IPC, 20I7b). El segundo tiene como propósito el desarrollo e implementación de una Estrategia Nacional de Clasificación, proporcionando recomendaciones para la Clasificación Nacional, incluyendo oportunidades de clasificación para los deportistas, la formación de clasificadores, 
y velar por el cumplimiento del Código de Clasificación de Deportistas a nivel nacional.

Cabe indicar aquí que, en los últimos años, hemos asistido a un intenso proceso de integración y/o independencia de modalidades deportivas paralímpicas, tradicionalmente al amparo de federaciones internacionales específicas para grupos de discapacidad (lesión medular, parálisis cerebral, discapacidad visual, discapacidad intelectual...), en su equivalente de deporte normalizado bajo el vocablo 'para' (e.g. para-triatlón sería la modalidad adaptada para personas con discapacidad dentro del triatlón). Este proceso ha creado en ocasiones ciertas disonancias entre la realidad internacional y la nacional, que motivan la necesidad de este trabajo en pro de poder analizar la situación actual en materia de clasificación y desarrollar una Estrategia Nacional de Clasificación, tal y como recomienda el Comité Paralímpico Internacional (IPC, 20I 7b). El desarrollo e implementación de la misma recae sobre el Comité Paralímpico Español (CPE), ente creado en 1995 con la misma naturaleza y funciones que las del Comité Olímpico Español a tenor de las modificaciones realizadas en la Ley del Deporte en I998. Desde su creación, el CPE se configuró como el órgano de unión y coordinación de todo el deporte para personas con discapacidad en el Estado español, con una estrecha colaboración con el Consejo Superior de Deportes. Concretamente, en el Artículo 48.6 de la Ley Io/I990, de I 5 de octubre, del Deporte, se estipula que "el Comité Paralímpico Español tiene la misma naturaleza y ejerce funciones análogas (...) respecto de los deportistas con discapacidades físicas, sensoriales, psíquicas y cerebrales. En atención a su objeto, naturaleza y funciones en el ámbito deportivo se declara de utilidad pública”.

La Guía sobre Clasificación de la Discapacidad en Deporte Paralímpico (Reina y VilanovaPériz, 20I6) recoge la estructura organizativa del deporte paralímpico, tanto nacional como internacional. No obstante, a principios de 2017 ya se han recogido cambios en la misma al independizarse del IPC los cuatro para-deportes de verano que hasta los Juegos Paralímpicos de
Rio estuvieron a su amparo: atletismo (World Para Athletics), levantamiento de pesas (World Para Powerlifting), natación (World Para Swimming) y tiro (World Shooting Para Sport). A ello debemos unir otros tantos deportes de invierno que harán su debut bajo una nueva estructura organizativa en los próximos Juegos Paralímpicos de Pyeongchang en 20I 8: esquí alpino (World Para Alpine Skiing), esquí nórdico y biatlón (World Para Nordic Skiing), snowboard (World Para Snowboard) y hockey en trineo (World Para Ice Hockey). Además de este cambio en el escenario internacional, encontramos:

a. Deportes paralímpicos que están al amparo de federaciones específicas por grupos de discapacidad:

- Ceguera y deficiencia visual: fútbol-5, goalball y judo, al amparo de la International Blind Sports Federation (IBSA).

- Usuarios de silla de ruedas y amputaciones: esgrima en silla de ruedas, al amparo de la International Wheelchair and Amputee Sports Federation (IWAS).

b. Deportes paralímpicos con su propia federación deportiva internacional, incluyendo: baloncesto en silla de ruedas (International Wheelchair Basketball Federation), boccia (Boccia International Sports Federation), fútbol- $7^{8}$ (International Federation of Cerebral Palsy Football), rugby en silla de ruedas (International Wheelchair Rugby Federation) y voleibol sentados (World Para-Volley).

c. Deportes paralímpicos integrados en federaciones específicas del deporte en cuestión: bádminton' (Badminton World Federation), ciclismo -ruta y pista- (International Cycling Union),

8. Presente en el programa de los Juegos Paralímpicos de Rio 20I6, pero no en los de Tokio 2020.

9. No presente en el programa de los Juegos Paralímpicos de Rio 20I6, pero si en los de Tokio 2020. 
curling -deporte de invierno- (World Curling Federation), hípica -modalidad de doma clásica- (Fédération Equestre Internationale), piragüismo (International Canoe Federation), remo (World Rowing Federation), taekwondo ${ }^{\text {Io }}$ (World

Taekwondo Federation), tenis de mesa (International Table Tennis Federation), tenis en silla de ruedas (International Tennis Federation), tiro con arco (World Archery), triatlón (International Triathlon Union) y vela $^{\text {II }}$ (World Sailing).

Aunque existe una plena equivalencia estructural a nivel nacional de los deportes expuestos en el apartado c) (e.g. piragüismo adaptado está integrado en la Real Federación Española de Piragüismo), no ocurre lo mismo con las modalidades deportivas de los epígrafes a) y b). Ello supone un gran reto organizativo y de optimización de recursos, frecuentemente escasos, que se destinan en España al deporte paralímpico, y al para-deporte por extensión. Por ejemplo, un deporte específico para personas con discapacidad como es la Boccia, que permite la participación de personas con seis deficiencias elegibles diferentes, a nivel nacional está representado por dos federaciones diferentes en función de la deficiencia presentada por el deportista: I) ataxia, atetosis o hipertonía, que concurriría al amparo de la Federación Española de Deportes para Personas con Parálisis Cerebral y Daño Cerebral Adquirido (FEDPC), o 2) deficiencia en la fuerza muscular (e.g. lesión medular), deficiencia del rango de movimiento pasivo (e.g. artrogriposis) o deficiencias en extremidades (e.g. amputaciones), que concurriría al amparo de la Federación Española de Deportes para Personas con Discapacidad Física (FEDDF). Además, este problema se acentúa en deportes que incluyen todas las deficiencias elegibles para deporte paralímpico, como es el caso del atletismo o la natación. Por ejemplo, atletismo incluye deportistas con las seis deficiencias elegibles expuestas para

Io. No presente en el programa de los Juegos Paralímpicos de Rio 20I6, pero sí en los de Tokio 2020.

I I. Presente en el programa de los Juegos Paralímpicos de Rio 20I6, pero no en los de Tokio 2020.
Boccia, a las que debemos unir la diferencia en la longitud de piernas y la baja estatura, la deficiencia visual -al amparo de la Federación Española de Deportes para Ciegos (FEDC)- y la intelectual -al amparo de la Federación Española de Deportes para Personas con Discapacidad Intelectual (FEDDI)-. El resultado no es otro que, mientras que a nivel internacional un determinado para-deporte está articulado por una única federación o estructura organizativa, a nivel nacional lo está por dos, cuatro, o hasta cinco federaciones si incluimos el deporte para personas con deficiencias auditivas, al amparo de la Federación Española de Deportes para Sordos (FEDS).

Debido a la intensa actividad internacional en este tópico, la aparición de nuevos documentos de obligado cumplimiento por parte de las federaciones internacionales de para-deporte a las que están adscritas las federaciones nacionales, y la reciente publicación de modelos de recomendación para la aplicación de políticas coordinadas en materia de clasificación a nivel nacional, los objetivos del presente estudio son: I) analizar la actividad y recursos que, en materia de clasificación, tienen las federaciones nacionales con responsabilidad en deportes paralímpicos, 2) evaluar el grado de conocimiento que las federaciones nacionales tienen sobre el nuevo Código de Clasificación de Deportistas y los Estándares Internacionales de IPC, y 3), establecer las bases de una Estrategia Nacional de Clasificación que sirva como hoja de ruta para la Comisión de Clasificación del Comité Paralímpico Español.

\section{Metodología}

\subsection{Participantes}

La población objeto de estudio son las diferentes federaciones nacionales que tienen responsabilidad en la promoción y organización de la competición de deporte paralímpico, ya sean específicas de grupos de discapacidad (polideportivas) o unideportivas, donde la 
modalidad de para-deporte está integrada en su estructura. Dado que los objetivos del estudio se circunscriben al grado de conocimiento y aplicación de documentos y políticas promovidas por el Comité Paralímpico Internacional, se invitó a participar en el estudio a todas aquellas federaciones nacionales con responsabilidad en deportes incluidos en los Juegos Paralímpicos, ya sean de verano (Rio 2016 y Tokio 2020) o invierno (Sochi 20I4 y Pyeongchang 20I8). Los responsables en materia de clasificación, o directores técnicos en su defecto, de las siguientes federaciones participaron de forma anónima en el estudio, aportando información sobre los diferentes deportes paralímpicos sobre los que tienen responsabilidad:

a. Federaciones específicas por grupos de discapacidad o polideportivas:

- Federación Española de Deportes para Ciegos (FEDC): atletismo, esquí alpino, esquí nórdico, fútbol-5, goalball, judo y natación.

- Federación Española de Deportes para Personas con Discapacidad Física (FEDDF): atletismo, baloncesto en silla de ruedas, boccia, esgrima, esquí alpino, esquí nórdico, natación, levantamiento de pesas, rugby en silla de ruedas, snowboard y voleibol sentados.

- Federación Española de Deportes para Personas con Discapacidad Intelectual (FEDDI): atletismo y natación.

- Federación Española de Deportes para Personas con Parálisis Cerebral y Daño Cerebral Adquirido (FEDPC): atletismo, boccia, esquí alpino, esquí nórdico, fútbol-7, natación, y snowboard.

b. Federaciones unideportivas: Federación Española de Bádminton (FEB), Real Federación Española de Ciclismo (RFEC), Real Federación Hípica Española (RFHE), Real Federación Española de Piragüismo (RFEP), Real Federación Española de Taekwondo, Real Federación Española de Tenis de Mesa (RFETM), Real Federación Española de Tenis (RFET), Real
Federación de Tiro con Arco (FETA) y Federación Española de Triatlón (FETRI).

Sólo no se ha podido obtener información de remo y vela, lo que supone una tasa de respuesta del $92,86 \%$ de los deportes y el $86,67 \%$ de las federaciones nacionales que eran objetivo de estudio.

\subsection{Instrumento y procedimiento}

Se ha realizado un cuestionario ad hoc para atender a los diferentes temas de interés para con el nuevo Código de Clasificación de Deportistas del IPC y los Estándares Internacionales relacionados. La elaboración del mismo se ha realizado siguiendo los siguientes cinco pasos:

I. Se contactó con seis clasificadores nacionales e internacionales de deportes paralímpicos como fútbol-7, boccia, atletismo y natación, y un para-deporte como slalom en silla de ruedas, de los cuales tres tenían experiencia en investigación en clasificación en deporte paralímpico. Se les pidió que elaborasen preguntas sobre cualquier aspecto que consideraran de interés para con la clasificación en deporte paralímpico. Se obtuvo una tasa de respuesta del $66 \%$ al recibir respuesta de cuatro de los seis clasificadores invitados para el focus group.

2. Todas las aportaciones o preguntas recogidas en la fase anterior se agruparon en cinco secciones temáticas, que se contrastaron con el primer borrador del Manual de Buenas Prácticas sobre Clasificación Nacional distribuido por el IPC, y así recoger todos los aspectos relevantes del mismo. La versión inicial del cuestionario constó de 23 preguntas.

3. Se envió el borrador del cuestionario al presidente de la Comisión de Clasificación ${ }^{12}$

I2. Profesor titular de universidad, con I 6 años de experiencia en clasificación a nivel nacional, y actualmente jefe de Clasi- 
del CPE, quien realizó modificaciones en las preguntas y categorías de análisis. Se mantuvo el número de categorías ( 5 ), pero se modificó la temática de algunas de ellas. En cuanto a las preguntas, se modificaron algunas y se añadieron otras, pasando a tener un total de $4 \mathrm{I}$ preguntas.

4. En la cuarta fase se contactó por correo electrónico con la Comisión de Clasificación del CPE, compuesta por seis componentes, además de la citada presidencia, del paso 3): dos trabajadores del CPE, una experta en clasificación a nivel internacional (ex-trabajadora de IPC) y profesora en actividad física y deporte adaptado, un médico y clasificador internacional para World Para Swimming, otro médico y clasificador internacional IBSA y una médico del deporte, también trabajadora externa para el CPE. A todos ellos se les hizo llegar el borrador del cuestionario para que aportaran posibles sugerencias y opiniones, recibiendo cinco respuestas al correo enviado $(83,3 \%)$ e incorporándose pequeñas modificaciones a la herramienta de recolección de datos.

5. En la quinta fase, los investigadores del presente trabajo se reunieron en Madrid con los dos componentes de la Comisión de Clasificación que son trabajadores del CPE. En esa reunión se realizó una puesta en común de todas las aportaciones recibidas, concretándose los últimos cambios de contenido y formato. Así, por ejemplo, en la versión inicial del cuestionario se utilizaban los términos 'para-deporte' e 'impedimento', de acuerdo con la nomenclatura que utiliza IPC (20I 5 b) y la CIF (OMS, 200I), siendo reemplazados por los términos 'deporte paralímpico’ y 'deficiencia' respectivamente, al ser términos que reconoce el CPE como oficiales y traducción oficial de la CIF al contexto español, respectivamente.

ficación para IFCPF, miembro del Comité de Clasificación de BISFed y clasificador internacional para World Para Athletics.
El documento final, de 42 ítems (Anexo I), contiene cinco áreas temáticas: I) introducción y cuestiones preliminares, 2) gestión de la clasificación a nivel organizativo y estructural, 3) gestión de la clasificación en competición,

4) formación y actualización de clasificadores y 5) otras cuestiones de interés.

El CPE envió una carta de invitación para participar en el estudio a todas las federaciones nacionales que tienen competencias en deporte paralímpico, quienes nos pusieron en contacto con el/la responsable en materia de clasificación. Previo al inicio de las entrevistas se realizó un estudio piloto, en el que uno de los investigadores entrevistó a un tercero, recibiendo feedback por parte del presidente de la Comisión de Clasificación del CPE. El proceso de recolección de datos se realizó en las tres siguientes fases:

I. Entrevistas realizadas vía teléfono o Skype por parte del equipo investigador. La posibilidad de utilizar la herramienta elaborada como entrevista semiestructurada permitía obtener cierto grado de flexibilidad en las respuestas de los entrevistados para expresar sus ideas, opiniones y actitudes (Sparkes, 2013). De acuerdo con Bernard (I988), este formato es el más utilizado cuando no se tiene la oportunidad de entrevistar a una persona más de una vez y cuando una serie de preguntas sobre un tópico determinado (clasificación en deporte paralímpico en nuestro caso) se pregunta a muchas personas, además de no permitir que quedaran preguntas sin responder. Sin embargo, por esta vía, se entrevistó a las federaciones de hípica (RFEH), piragüismo (RFEP), triatlón (FETRI), ciclismo (RFEC) y la FEDPC (Federación Polideportiva para Parálisis Cerebral y Lesión Cerebral). Esta fase se realizó entre mayo y junio de 2016.

2. Ante las dificultades de concertar una entrevista con los responsables en materia de clasificación o la no identificación de éstos en los organigramas de las federaciones nacionales, se remitió una 
segunda carta de invitación a participar en el estudio a aquellas federaciones nacionales que no habían contestado hasta la fecha. Transcribiendo las preguntas del guion de la entrevista a un documento de Google Docs, se obtuvieron respuestas de las federaciones polideportivas de discapacidad física (FEDDF), visual (FEDC) e intelectual (FEDDI), además de las de tiro con arco (FETA) y taekwondo. Esta fase se realizó entre octubre y noviembre de 2016 .

3. Tras la celebración de la jornada técnica sobre la Estrategia Nacional sobre la Clasificación de la Discapacidad en Deporte Paralímpico, celebrada en Madrid el 26 de noviembre de 2016 , se concretaron los contactos para completar la toma de datos con bádminton (FEB), tenis (RFET) y tenis de mesa (RFETM). Esta fase se realizó en diciembre de 2016 .

Todos los datos e identidades de los entrevistados fueron tratados de forma confidencial y siguiendo las directrices estatales sobre protección de datos personales.

\subsection{Análisis de resultados}

La transcripción de las entrevistas se realizó con la técnica verbatim (palabra a palabra) por parte del mismo investigador para dar consistencia al estudio (Bazeley y Jackson, 20I3). Las respuestas registradas por la vía cuestionario online (fases 2 y 3 ) se registraban en una matriz de contenido de Microsoft Excel, con el mismo número de celdas que los ítems utilizados en las entrevistas (fase I). Tanto la información obtenida vía entrevista como cuestionario fue analizada por la técnica de análisis de contenido (Hsieh y Shannon, 2005).

$\mathrm{Al}$ tratarse de un estudio de naturaleza cualitativa/descriptiva los datos se redujeron mediante análisis de frecuencias a través de SPSS (Statistical Package for Social Sciences, version 2I.o para Windows, SPSS Inc., Chicago, IL, USA).

\section{Resultados y discusión}

Debido a la naturaleza cualitativa del estudio, presentaremos los resultados atendiendo a las cinco áreas temáticas de análisis, seguido de la discusión de los mismos. Ello permitirá al lector seguir con más facilidad los hallazgos encontrados, atendiendo a diferentes criterios de rigor como (Lincoln y Guba, I985): credibilidad (i.e. haber involucrado a clasificadores nacionales e internacionales en el diseño de la herramienta de recolección de datos), transferibilidad (i.e. obtención de información de federaciones nacionales con diferente estructura y naturaleza: polideportivas $v s$. unideportivas), confirmación (i.e. análisis de contenido realizado por un investigador que no tenía relación alguna con las federaciones nacionales participantes o el propio CPE) y confianza (i.e. reclamando la participación de todas las federaciones nacionales hasta llegar a una tasa de respuesta del 92,86\% de los deportes paralímpicos entre 2014 y 2020).

\subsection{Introducción y cuestiones preliminares}

Todas las federaciones nacionales ofrecen alguna información sobre clasificación en su para-deporte. Sin embargo, existe una gran disparidad en cuanto a los criterios o formato de tal información, dificultando en ocasiones la localización de la misma o no estando actualizada a las últimas versiones. En cuanto a la localización, algunas federaciones enlazan al reglamento de su homónima internacional, especialmente en el caso de las federaciones polideportivas (e.g. FEDPC o FEDDF), ya que son varios los deportes a su amparo. Otras federaciones ubican la información sobre clasificación en la sección del comité médico (e. g. FEDC) o a un enlace externo del CPE (e. $g$. FEDDI). Salvo algunas excepciones $(e . g$. tiro con arco), no es frecuente la información permanente sobre formación en clasificación, y sólo taekwondo no ofrece información alguna en esta materia, deporte que hará su debut en los Juegos Paralímpicos de Tokio 2020. La 
Guía sobre Clasificación de la Discapacidad en Deporte Paralímpico (Reina y VilanovaPériz, 2016) trata de dar una respuesta a esta problemática, facilitando el acceso a la información sobre clasificación en las federaciones nacionales e internacionales (i.e. enlaces web y códigos QR).

Al preguntar a las federaciones unideportivas sobre si incluían algún contenido sobre clasificación en la formación de los técnicos o entrenadores de sus deportes, el $62,5 \%$ de las mismas informaron de que incluían información sobre la modalidad paralímpica en los bloques comunes de formación de técnicos, el $25 \%$ sólo informaba de la modalidad pero no sobre clasificación, y el I 2,5\% restante informaba de que estaba en desarrollo o en vías de implementarse. Se plasma pues una falta de uniformidad sobre los formatos o estrategias formativas que se aplican a nivel nacional con respecto a las que implementan, o deben hacerlo, las respectivas federaciones internacionales. La necesidad de uniformidad al respecto es crucial en los próximos años a tenor de los postulados del Código de Clasificación de Deportistas de IPC (20 I 5 a) y su modelo de buenas prácticas para la Clasificación Nacional (IPC, 20I 7b). El desarrollo de una adecuada Estrategia Nacional de Clasificación permitirá, entre otros aspectos que iremos analizando, que la información en materia de clasificación pueda llegar a las estructuras federativas y relacionadas con el deporte paralímpico a nivel autonómico, vivero real de futuros para-deportistas.

Al preguntarles sobre si conocían la existencia de un Código de Clasificación del IPC, de obligado cumplimiento por las federaciones internacionales, y por extensión las Nacionales, todas las federaciones contestaron afirmativamente, si bien algunas de ellas no estaban al tanto de una nueva versión del citado Código (IPC, 20I 5 a). En ese sentido, algunas federaciones manifestaron su dependencia total (e. g. taekwondo) sobre la información que les hacía llegar la federación internacional, y otra que tenía poca o ninguna información sobre las implicaciones de dicho Código para con la actividad de clasificación a nivel nacional
(FEDDI: $e$. g. clasificación basada en evidencias y específica de cada deporte).

\subsection{Gestión de la clasificación a nivel organizativo y estructural}

La primera indagación con respecto a esta categoría temática era analizar el tipo de personal que las federaciones nacionales tienen para el desempeño de responsabilidades en materia de clasificación. La mayoría de ellas tienen un colaborador externo al personal propio de la federación (46, I $5 \%$ ), dos federaciones unideportivas han designado responsables externos a la federación en materia de clasificación ( ( $5,38 \%$ ), una federación polideportiva tiene personal externo que recibe apoyo logístico de personal propio de la federación (7,69\%), el 23,08\% manifiesta que lo gestiona personal propio de la federación, y una federación unideportiva no especifica la necesidad de dicho personal (tenis -en silla de ruedas-). Descartando a las federaciones nacionales en las que la clasificación es gestionada por personal propio, el $50 \%$ de las restantes gratifica económicamente la labor del personal externo, mientras que el otro $50 \%$ no lo hace, siendo una labor totalmente voluntaria y ajena a su actividad profesional principal. A tenor de estos resultados y la información cualitativa obtenida al respecto, la remuneración por las labores en clasificación se circunscribe sólo al momento de la competición, donde ésta se lleva a cabo en el $84,62 \%$ de los casos. Se constata pues una gran labor de voluntariado en este ámbito, al igual que en otros ámbitos relacionados con la discapacidad (Morales et al., 2009; De Pablos, 2016) por lo que, ante las demandas internacionales en materia de clasificación, se hace preciso estimular la interacción entre las estructuras técnicas, médicas y de clasificación de las federaciones nacionales.

En cuanto a los recursos humanos existentes en la actualidad, existen tres perfiles básicos: médicos $(\mathrm{M})$, fisioterapeutas $(\mathrm{F})$ y técnicos deportivos $(\mathrm{T})$. Federaciones como la de triatlón $(\mathrm{M}+\mathrm{F}=7, \mathrm{~T}=7)$, ciclismo $(\mathrm{M}=5, \mathrm{~F}=5, \mathrm{~T}=3)$, 
piragüismo $(\mathrm{M}=\mathrm{I}, \mathrm{F}=3, \mathrm{~T}=3)$ y $\mathrm{FEDPC}^{\mathrm{I} 3}(\mathrm{M}$ $=4, \mathrm{~F}=5, \mathrm{~T}=9$ ) tienen una distribución más o menos homogénea entre tales perfiles o roles. Esto responde a los estándares internacionales sobre clasificadores (IPC, 20I6c), donde se diferencia entre personal sanitario (doctor, médico rehabilitador, fisioterapeuta, entre otros) y técnicos del deporte en cuestión (entrenadores, científicos del deporte, entre otros). Los primeros se encargarían de evaluar la presencia de una deficiencia elegible para ese deporte, así como el grado de severidad de la misma, mientras que los segundos evalúan el grado de impacto que ello tiene en la ejecución de las habilidades específicas del deporte (e. $g$. contribución del tronco, capacidad de agarre del aro o funcionalidad de los brazos en corredores en silla de ruedas en para-atletismo). Sin embargo, existe un grupo de federaciones en las que sólo cuentan con clasificadores con perfil sanitario (hípica $=5$, tiro con arco $=\mathrm{I}$, FEDDI = I -psicólogo- y FEDC = 2) o técnicodeportivo (tenis de mesa $=3$ ). En el caso de la FEDDF, dado el volumen de deportes que ésta gestiona, cuenta con clasificadores específicos para baloncesto en silla de ruedas $(\mathrm{n}=3)$, natación $(\mathrm{n}=3)$ y rugby en silla de ruedas ( $\mathrm{n}$ = 5), además de dos deportes no paralímpicos como hockey en silla de ruedas $(\mathrm{n}=4)$ y pádel $(\mathrm{n}=3)$. Taekwondo recurre a la federación internacional cuando precisa de clasificación, mientras que tenis en silla de ruedas (RFET) no tiene clasificadores en nómina. Esta disparidad en cuanto a perfiles y números se debe en parte a la evolución de la clasificación en deporte paralímpico, así como a la convivencia de estructuras federativas polideportivas y unideportivas. Además, se encuentra una falta de consenso acerca del tipo de certificación demandada para acreditarse como clasificador, aspecto que abordamos a continuación.

Al preguntar a las federaciones nacionales acerca del perfil que deberían tener los clasificadores de su deporte en los términos que estipula IPC (20I6c), el 30,77\% considera que debe conocer tanto la discapacidad como el deporte

I3. Tiene la responsabilidad de clasificación en atletismo y boccia en colaboración con la FEDDF. en cuestión (FEDPC, tiro con arco, tenis y tenis de mesa), el 53,85\% (piragüismo, ciclismo, triatlón, taekwondo, bádminton, y FEDDF) consideran que lo primordial es conocer la modalidad deportiva y las demandas para su práctica, y la $\operatorname{FEDC~}(7,69 \%)$ considera que debe ser personal médico especializado, o bien que la federación internacional sólo les permite ese perfil (hípica: 7,69\%). Observamos cómo aquellos deportes que se han incorporado más tarde al programa de los Juegos Paralímpicos o lo harán próximamente (i.e. triatlón y piragüismo en Rio 20I6, bádminton y taekwondo en Tokio 2020) u otros que están integrados en una federación unideportiva ( $e$. g. ciclismo) dan una gran importancia a los sistemas específicos del deporte, tal y como expusimos en la evolución de los sistemas de clasificación en deporte paralímpico (Hart, 20I7). Atendiendo a esta nueva realidad internacional y al enfoque de los sistemas de clasificación específicos de cada deporte y basados en evidencias, se hace preciso articular itinerarios formativos para clasificadores en deporte paralímpico, incluyendo criterios de obligado cumplimiento para la acreditación y su mantenimiento (IPC, 20I6c), tales como el perfil de acceso en el caso de los clasificadores técnicos (i.e. graduados en Ciencias de la Actividad Física y del Deporte, técnicos superiores en Actividades Físicas y Deportivas, técnicos deportivos), años de experiencia o número de deportistas clasificados.

Al preguntarles sobre el ofrecimiento o transmisión de información actualizada sobre los cambios e innovaciones en clasificación a sus clasificadores en activo, ésta se hace por diferentes vías: mail y/o web corporativa $(38,46 \%)$, reciclaje/re-acreditación de clasificadores (hípica y triatlón), acciones específicas por para-deporte (FEDDF), colaboraciones y/o acciones con la federación internacional equivalente (bádminton), mientras que el $30,77 \%$ restante no realiza acción alguna al respecto. Esta pregunta plasma nuevamente la disparidad de criterios utilizados para con la formación de clasificadores, en este caso en activo, siendo un aspecto crucial en un momento donde todas las federaciones internacionales 
deben adaptar sus reglas de clasificación al Modelo de Reglas de Clasificación recientemente aprobado por IPC (IPC, 20I7a) y de obligado cumplimiento antes del I de enero de 20 I 8. Estos datos tienen sendas ramificaciones importantes. Por un lado, se hace precisa una adecuada comunicación en materia de clasificación con las respectivas federaciones territoriales, ya que sólo el 30,77\% lo hace de oficio, una sólo lo hace si hay cambios, lo ubican en su página web $(\mathrm{n}=2)$ o directamente remiten a la web de la federación internacional $(\mathrm{n}=\mathrm{I})$, una sólo la envía si se la demandan, una sólo actúa en el caso de que haya clasificadores en activo, dos se coordinan con otras federaciones en materia de clasificación y una manifiesta que está en proceso de implementar medidas al respecto. Así, estos canales de transmisión de la información son importantes cuando se aplican medidas especiales de clasificación que no se recogen en competiciones internacionales. Sólo cuatro federaciones incluyen medidas especiales al respecto: hípica, que permite la participación de jinetes con perfiles funcionales más severos; piragüismo, que permite competir en la categoría $\mathrm{KL}_{3}$ otras deficiencias que no sean físicas; triatlón, que incluye una categoría para deportistas con discapacidad intelectual, y baloncesto en silla de ruedas, que permite la participación de mujeres en los equipos, aplicando una reducción de I punto al computar el número máximo de puntos de los cinco jugadores en pista $(<\mathrm{I} 4,5$ puntos, donde la puntuación de cada jugador/a va de I a 4.5 puntos, de menor a mayor funcionalidad). Es muy importante que todas estas medidas sean conocidas por todos los agentes implicados, especialmente en el momento del acceso o iniciación al para-deporte, ya que ayudarán a gestionar las expectativas de los practicantes y/o escoger la modalidad más adecuada a sus intereses o preferencias.

\subsection{Gestión de la clasificación en competición}

La gestión de la clasificación en competición es un tema de preocupación de las federaciones nacionales en varios frentes: costes, disponibilidad de clasificadores y atención a las demandas de la propia competición en un espacio corto de tiempo, ya que la clasificación suele realizarse justo antes del inicio de una competición. La primera pregunta abordada fue acerca del número de clasificadores y paneles disponibles en competiciones estatales. Sólo dos federaciones ( $5,38 \%$ ) consideran necesario la presencia de un mínimo de deportistas con necesidades de clasificación, ya sea porque tenga un estatus de deportista nuevo $(\mathrm{N})$ o en revisión (R). Aunque el estándar internacional sobre evaluación de deportistas (IPC, 20I6b) acepta paneles con dos clasificadores (personal sanitario y técnico), el 30,77\% de las federaciones nacionales articula paneles de tres componentes (médico, fisioterapeuta y técnico deportivo), con la posibilidad de que los componentes de la mesa se reduzcan a dos. Otro $46, \mathrm{I} 5 \%$ articula mesas de clasificación de dos componentes, el I $5,38 \%$ sólo un clasificador y una no dispone de clasificadores. Esta variabilidad en cuanto a la composición de las mesas se ve de nuevo en cuanto al número de mesas de clasificación, donde el 53,85\% sólo convoca una mesa por competición estatal, si bien el 38,46\% afirma que puede incrementarlo en caso de que el número de deportistas a clasificar exceda de un número determinado (e.g. > I 4,5) o se ofrezca la posibilidad de protesta ${ }^{14}$ en competición. El $30,77 \%$ restante sólo contempla la posibilidad de convocar un panel de clasificación, y una federación (tiro con arco) realiza la clasificación antes de la competición, no requiriendo de la convocatoria de clasificadores. Con respecto a las protestas, sólo el 46, I 5 \% tienen articulados protocolos de protesta, una federación sólo lo permite en caso de que haya dos paneles de clasificación, dos recogen la protesta pero es atendida a posteriori de la competición, una la remite a la federación internacional, y las tres restantes $(23,08 \%)$ no contemplan dicha posibilidad, estipulada claramente en el estándar internacional de protestas y apelaciones del IPC (20I6d).

I4. Proceso mediante el cual un club, equipo o deportista puede realizar una objeción formal a la clase asignada y, previo pago de un canon, pueda ser evaluado por una segunda mesa de clasificación. 
A tenor de estos datos y el contenido analizado de los entrevistados, existe una gran preocupación por la sostenibilidad económica de la clasificación en competición, dado que se deben asumir los costes de desplazamiento, alojamiento, manutención y per diem de los clasificadores, debiendo realizarla justo antes de la competición, con el impacto que ello conlleva en el desplazamiento de los competidores. Existe una gran variedad en cuanto a la contraprestación por los servicios prestados como clasificadores, oscilando entre los 80 y I $50 €$ por competición trabajada a sólo el abono del desplazamiento, alojamiento y manutención; aunque puede haber variaciones en función del volumen de deportistas a clasificar y la cualificación profesional o nivel del clasificador. Ninguna de las federaciones nacionales contempla además la figura del jefe de clasificación en competición o chief classifier (IPC, 20I6c), quien desempeñaría las labores de organización, resolución de dudas, articulación de posibles protestas y formación de nuevos clasificadores en prácticas, entre otras. Con los recursos destinados a la clasificación en la actualidad, no hay apenas posibilidades de protesta en competición, limitando los derechos de los deportistas a objetar sobre la clase asignada. Ello abre una puerta a la posibilidad de hacer la clasificación antes de la competición, tal y como realizan algunas federaciones, opción que recoge el nuevo Código de Clasificación de Deportistas (IPC, 20I 5a).

Ante la nueva posibilidad de poder llevar a cabo el proceso de clasificación fuera de la competición propiamente dicha, el 6I,54\% de las federaciones nacionales está de acuerdo con ello, dos creen que "debería" o "sería necesario”, dos no están de acuerdo y una muestra dudas acerca de la viabilidad de dicha opción. Pero la forma o procedimientos para llevarla a cabo son muy variados, esgrimiendo razones como sólo en el caso de hacer una consulta previa a la competición, que sea realizada sólo por parte de grupos o personas debidamente acreditadas, realizarse sólo en el caso de que luego pueda completarse el proceso de clasificación en competición o, simplemente, porque es más operativo, abarata costes y ayuda al desarrollo de la clasificación a nivel autonómico. Además, se presenta como una posibilidad de resolver protestas que no han podido atenderse en competición, especialmente en el caso de que sólo hubiera una mesa de clasificación.

A la hora de gestionar el proceso de clasificación, el 92,3 I \% de los entrevistados/ encuestados consideran que los clasificadores deben conocer cómo se organiza la competición de su para-deporte, con respuestas que incluyen "sí”, "imprescindible”, "por supuesto" o “esencial”. El mismo porcentaje considera que los clasificadores convocados a una competición deben, independientemente de su perfil o rol en la mesa, tener un adecuado manejo de las fichas de clasificación. Sólo una federación considera que al menos uno de los dos clasificadores debe conocer el deporte, y que sería "recomendable" que ambos conocieran las fichas de clasificación. Este alto porcentaje constata el grado de acuerdo respecto a la necesidad de que los dos o tres componentes de las mesas de clasificación complementen sus roles. Así, por ejemplo, mientras que el personal sanitario está evaluando el grado de espasticidad de un deportista con hipertonía por parálisis cerebral, un técnico deportivo debería estar cumplimentando en la ficha de clasificación los hallazgos encontrados. En contraposición, cuando el técnico lidera la fase de evaluación técnica, será el personal sanitario el que debe cumplimentar las fichas de clasificación. Y al preguntarles acerca del tipo o modelo de ficha de clasificación utilizada, el 23,08\% afirma utilizar la misma ficha de clasificación que su homónimo internacional, I 5,38\% las ha traducido literalmente, el 23,08\% las han adaptado, el I $5,38 \%$ tienen sus propias fichas de clasificación y el 23,08\% restante utiliza otros procedimientos o no tiene fichas de clasificación para llevar a cabo el proceso de clasificación. Unido al hecho de que el $76,92 \%$ afirma que sus fichas de clasificación siguen las directrices de su federación internacional, y actualiza las mismas con regularidad, constatamos la tendencia hacia fichas y procedimientos de evaluación de cada para-deporte, reto al que deben enfrentarse especialmente las federaciones polideportivas, con varios deportes en su seno. Así, federaciones 
como la FEDPC o FEDDF han pasado en los últimos años de tener fichas generales de su grupo de discapacidad, a tener fichas de clasificación en colaboración para deportes como para-atletismo, para-natación, boccia o slalom en silla de ruedas, deportes que incluyen deportistas susceptibles de tener licencia con una de las dos federaciones.

\subsection{Formación y actualización de clasificadores}

En cuanto a la formación de los clasificadores, encontramos que sólo el 53,85\% oferta o tiene programas formativos para nuevos clasificadores. Las últimas formaciones realizadas datan de entre 20I4 y 20I5, especificando algunas de ellas que se hace en colaboración con las federaciones autonómicas (e.g. triatlón), o federaciones polideportivas (e.g. FEDPC y FEDDF) que realizan sus formaciones diferenciando entre para-deportes. De hecho, FEDPC y FEDDF son las únicas federaciones nacionales que colaboran entre ellas en materia de formación de clasificadores. En cambio, al preguntarles si la formación que realizan se hace en colaboración con la federación internacional correspondiente, sólo piragüismo y bádminton afirman que así es, demostrando nuevamente que aquellos deportes que se han incorporado más tarde al programa de los Juegos Paralímpicos se acercan a los estándares internacionales estipulados por IPC, y de obligado cumplimiento por las federaciones internacionales.

El 53,84\% de las federaciones entrevistadas afirma controlar el perfil o formación previa de los nuevos clasificadores, aspecto que viene recogido en el estándar internacional sobre clasificadores (IPC, 20I6c), y sólo el 38,46\% del total $(83,33 \%$ de las que tienen oferta) tienen estipulado algún criterio sobre el perfil de los profesores que imparten dichas formaciones, preferentemente clasificadores internacionales o con una dilatada experiencia en el deporte paralímpico en cuestión. Una vez expuesta la panorámica general sobre la oferta en la materia que nos ocupa, a continuación se detallan las respuestas más relevantes sobre la acreditación o re-acreditación de tales clasificadores:
- En cuanto a la pregunta de si disponen de un programa de prácticas para completar la formación en clasificación, sólo la FEDPC, ciclismo, hípica y bádminton disponen de protocolos al respecto $(e . g$. rúbrica de evaluación de competencias, debilidades $v s$. fortalezas).

- $\quad$ El 46,I $5 \%$ de las federaciones nacionales dispone de algún criterio mínimo para que un clasificador en prácticas pueda ser clasificador nacional, con respuestas que oscilan desde 2 (bádminton), 5 (FEDC e hípica), a Io-I 5 (FEDPC).

- Sólo dos federaciones nacionales tienen un máximo estipulado de clasificadores en prácticas en competición (FEDPC $=3-4$, ciclismo $=2-3$ ).

- Sobre la re-acreditación de los clasificadores en activo, el 6I,54\% afirman tener alguna medida al respecto (i.e. seminarios de reciclaje, envío de información): anualmente (triatlón, ciclismo), cada dos años (bádminton, hípica, tiro con arco), o cuando hay cambios en las normativas internacionales (FEDPC, FEDDF, FEDC).

- Ante la última pregunta acerca de la idoneidad de que la formación en materia de clasificación pudiera ser online, sólo una federación está de acuerdo con ello, el 46, I 5 \% estaría sólo de acuerdo en hacer una introducción a la misma o la exposición de los elementos transversales básicos, otro $38,46 \%$ se opone a ello, defendiendo que toda la formación debe ser presencial debido al carácter técnico y práctico de la misma, y una última federación no se manifiesta al respecto.

\subsection{Otras cuestiones de interés}

El Comité Paralímpico Español aprobó en 2015 la constitución de una Comisión de Clasificación, precisamente en el momento en el que se estaba culminando el proceso de revisión por parte de las federaciones internacionales y los comités 
paralímpicos nacionales del nuevo Código de Clasificación de Deportistas (IPC, 201 5a) y los Estándares Internacionales relacionados con la clasificación en deporte paralímpico (IPC, 20I6a; IPC, 20I6b; IPC, 20I6c; IPC, 20I6d; IPC, 20I6e). Al preguntar a las federaciones nacionales sobre si conocían la existencia de dicha Comisión de Clasificación del CPE, el 53,85\% contestó afirmativamente y el 46, I $5 \%$ restante que no. Entre las funciones que tales federaciones nacionales consideran que debería tener la Comisión de Clasificación del CPE destacan la resolución o preparación de casos complejos o límite antes de asistir a competiciones internacionales, la actualización en materia de clasificación para adecuarse a los estándares internacionales, servir de nexo de unión para con la aplicación de las políticas establecidas por el IPC, ayudar al desarrollo de la clasificación en su deporte con especial atención al ámbito territorial, asistir en la formación de nuevos clasificadores o reacreditación de los que están en activo, asistir durante el proceso de protestas en/tras las competiciones internacionales, o diseñar una Estrategia Nacional de Clasificación, entre otras. Con respecto al diseño e implementación de una Estrategia Nacional de Clasificación, los entrevistados/encuestados se muestran a favor en un $69,23 \%$, mientras que una federación nacional no lo está, dos manifiestan que depende de la ascendencia que tenga sobre su autonomía en materia de clasificación, y una no se pronuncia al respecto. Los puntos que debería abordar dicha Estrategia serían, pero no limitados a:

- Homogeneizar los estándares internacionales de clasificación a nivel nacional, tal y como recoge el Modelo de Reglas (IPC, 20I7a).

- Aumentar los estándares de calidad durante los procesos de clasificación, respondiendo a la creciente profesionalización del deporte paralímpico de alto nivel (i. e. Plan ADOP).

- Establecer unas directrices comunes en materia de clasificación, respondiendo así al Modelo de Buenas Prácticas en Clasificación Nacional (IPC, 20I7b).
- Optimizar los procesos formativos de clasificadores, ajustándose al estándar internacional de formación y acreditación de clasificadores en deporte paralímpico (IPC, 20I6c).

- Asesoramiento en la gestión de casos, dado que el CPE es en ocasiones el interlocutor oficial en deportes que integran múltiples deficiencias elegibles, pero que a nivel nacional están al amparo de diferentes federaciones polideportivas (e.g. atletismo, natación). En relación a este punto, algunas federaciones polideportivas consideran de utilidad esta colaboración.

Finalizamos este apartado de resultados y discusión, exponiendo una serie de puntos independientes, pero de interés para con los objetivos del presente trabajo:

- Al peguntarles por la posibilidad de elaborar una red de clasificadores a nivel nacional a fin de discutir temas de interés, el $84,62 \%$ de las federaciones nacionales se mostró a favor de la misma.

- $\quad$ Al preguntarles acerca de si los deportistas y técnicos de su deporte valoran adecuadamente la clasificación deportiva, existe una gran disparidad de respuestas al respecto: el 30,77\% consideran que "sí", el I 5,38\% consideran que "no", "depende" o no se pronuncian al respecto, y el $23,08 \%$ restante consideran que es un tema "problemático", tal y como se ha constatado en varios medios durante los últimos Juegos Paralímpicos de Rio 2016 (Davies, 2016).

- Finalmente, dado que IPC insta a las federaciones internacionales al desarrollo de sistemas de clasificación basados en la investigación y el estudio de la relación entre el grado de deficiencia y el impacto que tiene en el rendimiento de un determinado para-deporte, el Ioo \% de las federaciones nacionales se mostraron accesibles a colaborar para el desarrollo de acciones en la materia. Así, algunas 
federaciones nacionales ya colaboran en mayor o menor medida en la realización de investigaciones propias o en colaboración en la materia (i.e. boccia, fútbol-7, paratriatlón, slalom en silla de ruedas).

\section{Conclusiones}

En el apartado de "Resultados y discusión" hemos abordado dos de los objetivos de la presente investigación: I) analizar la actividad y recursos que, en materia de clasificación, tienen las federaciones nacionales con responsabilidad en deportes paralímpicos, y 2) evaluar el grado de conocimiento que las federaciones nacionales tienen sobre el nuevo Código de Clasificación de Deportistas y Estándares Internacionales del IPC. En este último epígrafe trataremos de dar respuesta al tercer objetivo planteado, que no es otro que establecer las bases de una Estrategia Nacional de Clasificación que sirva como hoja de ruta para la Comisión de Clasificación del Comité Paralímpico Español y las diferentes federaciones nacionales con modalidades paralímpicas o de para-deporte:

- Se hace preciso intensificar la comunicación entre las federaciones nacionales, especialmente aquellas que comparten deportes por su naturaleza de federación polideportiva. La imposición de estándares internacionales en materia de clasificación recomienda el trabajo en red entre todos aquellos técnicos y profesionales relacionados, contribuyendo a la optimización del tiempo y los recursos económicos destinados al respecto.

- Estrechar la colaboración entre aquellas federaciones nacionales que compartan deficiencias elegibles para su deporte. El estándar internacional sobre deficiencias elegibles en deporte paralímpico (IPC, 20I6a) concreta la denominación de las deficiencias elegibles y las condiciones de salud que subyacen a las mismas (e. g. una parálisis cerebral conllevará una deficiencia elegible de hipertonía, ataxia o atetosis; mientras que una lesión medular conllevará una deficiencia elegible de falta de fuerza muscular por debajo del nivel de la lesión). En el Fórum de Investigación en Clasificación desarrollado por IPC en febrero de 2017 se mostraron protocolos estandarizados para la evaluación de las deficiencias elegibles (i.e. visual, intelectual y física), por lo que no es de extrañar que en los próximos años estos protocolos sean comunes a todos los deportes paralímpicos.

- Existe una demanda en cuanto a la formación, no sólo de clasificadores, sino también de iniciar o dar a conocer los aspectos básicos sobre clasificación a los técnicos deportivos. La inclusión de contenidos sobre clasificación en los bloques comunes de los programas formativos oficiales de técnicos deportivos ayudaría a que cualquier técnico de un deporte pudiera iniciar en la práctica deportiva a una persona con discapacidad, ayudando a la detección temprana de potenciales deportistas y a una correcta gestión de las expectativas de logro. En este sentido, el CPE realizó en abril de 2017 una formación transversal en clasificación, a la que fueron invitados responsables técnicos de todas las federaciones nacionales.

- Se requiere de una adecuada formación práctica en materia de clasificación, dado el carácter instrumental de la misma (e. g. evaluación de la fuerza muscular, espasticidad, reflejos alterados, etc.), suponiendo un reto el hacer llegar la clasificación a las federaciones territoriales, donde realmente acceden los nuevos deportistas que requieren de clasificación hasta que ésta pueda ser confirmada en instancias superiores (i. e. nivel nacional o internacional).

- Relacionado con el punto anterior, existe la necesidad de un mayor compromiso a largo plazo de los clasificadores en activo ya que, debido al marcado carácter 
voluntario de esta labor y la insuficiente o nula remuneración económica, hay una gran fluctuación en número y perfiles de clasificadores.

- Se recomienda involucrar a universidades y/o centros educativos en clasificación. Las primeras pueden hacer una gran labor en cuanto a colaborar en investigación e incrementar el prestigio o posicionamiento internacional de nuestro país o determinados deportes, además de poder acercar la clasificación a sus aulas, especialmente en formaciones relacionadas como Ciencias de la Actividad Física y del Deporte, Medicina o Fisioterapia. Los segundos, durante la impartición de los bloques comunes y/o específicos de los títulos de técnicos deportivos, podrían incluir contenidos de clasificación, especialmente en los módulos comunes de enseñanza deportiva en "Actividad Física Adaptada y Discapacidad”, ya sea en el ciclo inicial (MED-CIO3) o final (MED-C203).

- $\quad$ Se requiere de bases de datos ágiles, que permitan intercambiar información entre federaciones territoriales y nacionales, y entre nacionales e internacionales. En cualquier caso, el intercambio de información debe estar sujeto al nuevo estándar internacional sobre protección de datos (IPC, 20I6e), así como la legislación vigente sobre protección de datos personales, ya que se trabaja con información sensible que incluye historias clínicas de los deportistas para acreditar su deficiencia elegible.

- Existe una demanda de apoyo económico para el desarrollo de políticas y acciones en materia de clasificación, de ahí el marcado carácter de voluntariado existente. Cabe destacar la escasa presencia del deporte paralímpico en los medios de comunicación, con la consiguiente dificultad para conseguir apoyos o patrocinios por el insuficiente retorno que ello conlleva. La responsabilidad social corporativa
(RSC), también llamada responsabilidad social empresarial (RSE) o inversión socialmente responsable, es un mecanismo a explotar debido a la contribución activa y voluntaria para con la mejora social, tratando de asociar los valores del Movimiento Paralímpico (Spirit in Motion) como valor añadido de potenciales empresas colaboradoras.

- Se requiere de una adecuada colaboración entre aquellas federaciones polideportivas que expiden licencias a deportistas para un mismo para-deporte. Esta colaboración en materia de clasificación permitiría optimizar los recursos destinados a un mismo deporte.

Tras la finalización de la jornada sobre la Estrategia Nacional de Clasificación en Deporte Paralímpico (Madrid, 26 de noviembre de 20I6), y a la que asistieron representantes del roo \% de las federaciones nacionales participantes en el presente estudio, además de los miembros de la Comisión de Clasificación del CPE, emergieron una serie de medidas adicionales a las anteriormente planteadas:

- Articular una red de trabajo a nivel territorial para la elegibilidad de nuevos para-deportistas. Esta medida permitiría abaratar costes para con la convocatoria de mesas de clasificación regionales, cuyas decisiones deben ser ratificadas a nivel nacional.

- Establecer un protocolo que ayude a las federaciones nacionales en la preparación de documentación y material complementario de deportistas que acudan a competiciones internacionales. Esta medida es de vital importancia en aquellos casos que se encuentren en el límite de dos clases o para el cumplimiento de la deficiencia mínima para ser elegible.

- Establecer un protocolo de actuación y comunicación para la gestión de protestas en competiciones internacionales, proveyendo directrices para una correcta 
presentación de las mismas, suministrando información o pruebas de apoyo.

- Establecer un protocolo para la solicitud de re-clasificación de un deportista por razones médicas (Medical Request), aplicable cuando la deficiencia elegible ha podido variar o fluctuar (e.g. empeoramiento en la función muscular por esclerosis múltiple).

- Favorecer la colaboración entre federaciones para con aquellos clasificadores (i.e. médicos y fisioterapeutas) que pudieran actuar en diferentes para-deportes.
- Habilitar un espacio de comunicación en la web del Comité Paralímpico Español, con acceso a documentos relevantes sobre clasificación del IPC, así como aquellos documentos producidos por la propia Comisión de Clasificación del CPE (e.g. directorio de contactos).

- Involucrar a las federaciones nacionales en la elaboración de nuevos documentos ( $e$. g. mínimas deficiencias elegibles en cada deporte paralímpico) o actualización de la Guía de Clasificación de la Discapacidad en Deporte Paralímpico (Reina y VilanovaPériz, 2016). 
Anexo 1. Análisis de la situación sobre la clasificación en deporte paralímpico en España. Hacia una Estrategia Nacional de Clasificación

- Introducción y cuestiones preliminares:

I. ¿Tiene su federación nacional una práctica deportiva para personas con discapacidad o modalidad de deporte paralímpico?

- En caso de que sean varios deportes, por favor, indique cuáles son.

2. La página web de su federación, ¿̇ofrece algún tipo de información sobre deporte paralímpico?

- En caso afirmativo, ¿hay alguna información específica sobre clasificación?

3. En caso de tratarse de una federación unideportiva, ¿se incluye algún contenido de clasificación en la formación de técnicos o entrenadores de su deporte?

4. ¿Sabía que el Comité Paralímpico Internacional tiene un Código de Clasificación que deben cumplir todas las federaciones internacionales de deportes paralímpicos, y por extensión sus respectivas federaciones nacionales?

- En caso afirmativo, ¿sabía que ese Código ha sido recientemente actualizado y se esperan cambios en algunos sistemas y procesos de clasificación en los deportes paralímpicos?

5. ¿Está su federación al corriente de los sistemas de clasificación existentes que hay en su modalidad deportiva paralímpica a nivel internacional?

- Sobre la gestión de la clasificación en su federación nacional:

6. ¿Existe una persona en su federación nacional que tenga competencias asignadas en la gestión de la clasificación?
- En caso afirmativo, ¿es un trabajador/a de la federación o un colaborador/a externo?

- Y en caso de que sea un colaborador/a externo, ¿̇recibe éste algún tipo de gratificación o reconocimiento a su labor?

7. ¿Cuántos clasificadores en activo dispone su federación?

- En caso afirmativo, ¿podría especificar el perfil de los mismos?

8. El Comité Paralímpico Internacional discrimina entre dos tipos de clasificadores: médico y técnico. Los primeros se encargan de determinar la elegibilidad del deportista y la severidad de su deficiencia (e.g. médicos o fisioterapeutas), mientras que los técnicos evalúan el impacto que ello tiene en hacer las habilidades propias del deporte en el que va a competir:

- ¿Cuál cree que debería ser el perfil ideal de los clasificadores técnicos?

9. ¿Ofrece su federación nacional información actualizada sobre los cambios e innovaciones en clasificación en su modalidad de deporte paralímpico a sus clasificadores en activo?

- En caso afirmativo, ¿por qué medio, vía y/o frecuencia?

ıо. ¿Intercambia su federación nacional información de interés sobre clasificación con otras federaciones nacionales?

I I. ¿Suele su federación nacional trasladar la información que tiene en materia de clasificación a sus federaciones territoriales, si las hubiere?

I2. ¿Tiene su federación nacional alguna medida especial o contradictoria con respecto a la clasificación, y que no se 
recoja a nivel de competición internacional? (e. g. tener una medida de discriminación positiva, dando un punto menos, a la clasificación de una jugadora en un equipo de baloncesto en silla de ruedas).

- Sobre la gestión de la clasificación en competición:

I3. ¿Existe un número mínimo de deportistas a clasificar en competición para poder convocar una mesa o panel de clasificación?

- En caso afirmativo, ¿cuántos?

I4. ¿Cuántos clasificadores como mínimo cree que debería tener una mesa de clasificación?

- Indique una relación priorizada de los perfiles de tales componentes.

I 5. ¿Cuántas mesas de clasificación cree que debería haber en una competición nacional?

I6. ¿Sería apropiado que todos los componentes de la mesa de clasificación tuviesen nociones básicas sobre el deporte a clasificar?

I7. ¿Sería apropiado que todos los componentes supieran cómo cumplimentar los diferentes apartados de la ficha de clasificación?

I 8. ¿Qué opinión tiene de las fichas de clasificación que utiliza en la actualidad su deporte?

19. ¿Utiliza su federación nacional una ficha y procedimientos similares a los que utiliza su federación internacional?

- Tanto en caso positivo como negativo, justifique su respuesta.

20. ¿Está su federación nacional al tanto de los posibles cambios que se pueden dar en los sistemas y procesos de clasificación en su modalidad de deporte paralímpico?

- En caso afirmativo, ¿cuáles son los pasos para actualizar las normas o acciones a nivel nacional?
2I. Los clasificadores que asisten a una competición, ¿̇reciben algún tipo de remuneración o reconocimiento por su labor como oficiales de competición?

- En relación a la pregunta anterior, ¿cuál piensa que debería ser la remuneración mínima por esta labor?

22. El IPC, en su nuevo Código de Clasificación, permite la realización de clasificaciones fuera de la competición, ¿cree que sería adecuado la realización de los procesos de clasificación no en el día justo antes de la competición?

- En caso afirmativo, ¿indique posibles alternativas a ello?

23. ¿Permite su federación nacional la posibilidad de protestar por parte de un deportista o club el resultado de una clasificación?

- En caso afirmativo, ¿podría describir brevemente el proceso seguido para ello?

- Sobre la formación y actualización de los clasificadores:

24. ¿Oferta su federación cursos de formación de clasificadores?

- En caso afirmativo, ¿cuándo fue la última vez que ofertaron uno?

- ¿Cuáles son los medios de difusión de la citada oferta?

25. ¿Colabora su federación nacional u obtiene recursos de la federación internacional para realizar cursos de clasificación?

- En caso afirmativo, ¿qué tipo de ayuda reciben?

26. ¿Oferta su federación nacional cursos de formación de clasificadores en colaboración con otras federaciones nacionales?

- En caso afirmativo, indicar cuáles y/o en qué deportes paralímpicos.

27. ¿Tiene su federación nacional alguna estrategia o mecanismo de control del 
perfil de las personas que desean acceder a realizar un curso de clasificación en su modalidad de deporte paralímpico?

- En caso afirmativo, ¿podría especificarlas brevemente?

28. ¿Qué perfil tienen los profesores o ponentes que dan los cursos de clasificadores en su federación nacional?

29. Cuando acaba una formación de clasificadores, ¿tiene su federación nacional un programa de prácticas para completar la formación?

- En caso afirmativo, ¿hay un tiempo máximo para poder completar las prácticas?

- ¿Disponen de alguna hoja o rúbrica de evaluación de las competencias del futuro clasificador/a para evaluar el dominio de sus competencias?

30. ¿Cuántas competiciones o número de deportistas debería realizar como mínimo un clasificador para poder ser clasificador nacional?

3I. ¿Tiene su federación un mínimo o un máximo de clasificadores en prácticas que permitiría asistir a realizar sus prácticas en competición?

- En caso afirmativo, ¿cuántos?

32. ¿Considera que sus clasificadores en activo deberían realizar un reciclaje de sus conocimientos y competencias en clasificación?

- En caso afirmativo, ¿cada cuánto tiempo?

33. ¿Cree que sería efectiva una formación de clasificadores sólo en formato online?

- Otras cuestiones de interés:

34. ¿Sabía que el Comité Paralímpico Español había constituido una Comisión Nacional de Clasificación en Deporte Paralímpico?

- ¿Cuáles piensa que podrían ser las funciones de esta Comisión Nacional de Clasificación y/o cómo cree que podría ayudar a las federaciones nacionales en esta materia?

35. ¿Cree que sería de utilidad disponer de una red de clasificadores que estuviesen comunicados a nivel nacional a fin de discutir temas de interés en la materia?

36. Tal y como recoge el IPC en su último Código de Clasificación, ¿considera necesaria la elaboración de un Plan Nacional de Clasificación?

- En caso afirmativo, ¿en qué debería contribuir a su labor como federación nacional?

37. ¿Tiene su federación nacional un código de buena conducta por parte de sus clasificadores en activo?

- ¿Lo considera necesario?

38. ¿Creen que los deportistas y técnicos valoran la clasificación deportiva en su deporte/s paralímpico/s?

39. Tal y como recoge el IPC en su nuevo Código de Clasificación en Deporte Paralímpico, se estimula a los deportistas a participar en investigaciones para mejorar los sistemas de clasificación, de manera que sean específicos de cada deporte paralímpico y basados en pruebas científicas: ¿estaría su federación nacional dispuesta a colaborar en algunas iniciativas que le pudieran proponer en este sentido?

40. ¿Qué alternativas creen que serían útiles para mejorar el estado de la clasificación nacional?

4I. ¿Estaría interesada su federación nacional en asistir a una sesión informativa promovida por la Comisión de Clasificación del Comité Paralímpico Español, prevista para noviembre de 2016 ?

42. ¿Alguna aportación adicional?

Muchas gracias por su colaboración 
Referencias bibliográficas

Bazeley, P. y Jackson, K. (eds.) (2013): Qualitative data analysis with NVivo. London: Sage Publications Limited.

Bernard, H. R. (I988): Research methods in cultural anthropology. Newbury Park, CA: Sage.

Brittain, I. (20I0): The paralympic games explained. Oxon: Routledge.

Davies, G. A. (4 de septiembre de 2016): Paralympic classification expert claims "cheating is not endemic" (en línea). $<$ http://www.telegraph.co.uk/paralympicsport/2016/o9/o7/paralympic-classificationexpert-claims-cheating-is-not-endemic $>$, acceso I 8 de febrero de 2017.

De Pablos, J. F. G. (2016): “El voluntariado deportivo". Revista Aranzadi de derecho de deporte y entretenimiento, 50: 87-106.

DePauw, K. P. y Gavron, S. J. (2005): Disability sport ( $2^{\text {nd }}$ Edition). Champaign, IL: Human Kinetics.

España. Ley Io/I990, de I 5 de octubre, del Deporte, Boletín Oficial del Estado, 6 de noviembre de I990, núm. 249, pp. 30397304II.

Hart, A. (inédito): "IPC's Head of Classification Meeting Introduction”. En 2017 Head of Classification Meeting. Ponencia llevada a cabo en Bad Honnef, Alemania.

Hsieh, H. F. y Shannon, S. E. (2005): “Three approaches to qualitative content analysis". Qualitative health research, I 5 (9): I 277-I 288.

IPC (20I7a): Classification Model Rules for Para Sport (en línea). <https://www.paralympic.org/ classification/20I 5-athlete-classification-code>, acceso I7 de febrero de 2017.

IPC (20I7b): Model of Best Practice on National Classification (en línea). <https://www. paralympic.org/classification/20I 5-athleteclassification-code>, acceso I 7 de febrero de 2017.
IPC (2016a): International Standard for Classifier Personnel and Training (en línea). <https:// www.paralympic.org/sites/default/files/ document/I6IO04I 500597I2_20I6_IO_O4_ International_Standard_for_Classifier_ Personnel_and_training_o.pdf>, acceso Io de febrero de 2017.

IPC (2016b): International Standard for Athlete Evaluation (en línea). <https:// www.paralympic.org/sites/default/files/ document/I6IO04I359I8977_20I6_IO_O4_ International_Standard_for_Athlete_ Evaluation.pdf>, acceso Io de febrero de 2017.

IPC (20I6c): International Standard for Eligible Impairments (en línea). <https:// www.paralympic.org/sites/default/files/ document/I 6IO04I45727I29_20I6_IO_O4_ International_Standard_for_Eligible_ Impairments_I.pdf $>$, acceso Io de febrero de 2017.

IPC (2016d): International Standard for Protests and Appeals (en línea). <https:// www.paralympic.org/sites/default/files/ document/I6I004I 45006926_20I6_IO_O4_ International_Standard_for_Protests_and_ Appeals.pdf>, acceso Io de febrero de 2017.

IPC (20I6e): International Standard for Classification Data Protection (en línea). $<$ https://www.paralympic.org/sites/default/files/ document/I6I004I44IO3299_20I6_IO_O4_ International_Standard_for_Classification_ Data_Protection.pdf $>$, acceso Io de febrero de 2017.

IPC (20I 5a): International Paralympic Committee Athlete Classification Code (en línea). <https:// www.paralympic.org/sites/default/files/docume nt/I 5 I 2 I 8 I 23255973_20I 5_I2_I 7\% 2BClassif ication\% $\%$ BCode_FINAL_o.pdf $>$, acceso ro de febrero de 2017.

IPC (20I 5b): Strategic Plan 2015 to 2018 . Strategic outlook for the International Paralympic Committee (en línea). <https://www. paralympic.org/sites/default/files/document/I 
506I9I33600866_20I 5_06+IPC+Strategic+ Plan+20I 5-20I 8_Digital.pdf>, acceso I 3 de febrero de 2017.

IPC (2007): International Paralympic Committee Classification Code (en línea). <https://www. paralympic.org/2007-classification-code>, acceso ro de febrero de 2017.

Lincoln, Y. S. y Guba, E. G. (I985): Naturalistic Inquiry (Vol. 75). Thousand Oaks, CA: Sage Publications.

Morales, F. M. et al. (2009): "Eficacia de una intervención para incrementar apoyo social en adolescentes discapacitados motores a partir del voluntariado de estudiantes de educación secundaria". Revista latinoamericana de psicología, 4I (I): I4 I-I 50.

OMS (200I): Clasificación Internacional del Funcionamiento, de la Discapacidad y de la Salud (en línea). <http://apps.who.int/iris/ bitstream/I0665/43360/I/924I 545445_spa. pdf $>$, acceso 5 de febrero de 2017.

OMS (I980): International Classification of Impairments, Disabilities, and Handicaps (en línea). <http://apps.who.int/iris/ bitstream/I0665/4I003/I/924I54 I 26I_eng. pdf $>$, acceso 5 de febrero de 2017 .

Paciorek, M. J. (20I I): “Adapted sport”, en Winnick, J. P. (ed.): Adapted physical education and sport. Champaign, IL: Human Kinetics.
Reina, R. y Vilanova-Périz, N. (2016): Guía sobre clasificación de la discapacidad en deporte paralimpico. Elche: Limencop S. L.

Reina, R. (20I4a): "Inclusión en deporte adaptado: dos caras de una misma moneda". Psychology, society and education, 6 (I): 55-67.

Reina, R. (20I4b): "Evidence-based classification in paralympic sport: application to football-7a-side". European journal of human movement, 32: I6I-I 85 .

Sanz, D. y Reina, R. (20I2): Actividades físicas $y$ deportes adaptados para personas con discapacidad. Badalona: Paidotribo.

Sparkes, A. C. y Smith, B. (20I3): Qualitative research methods in sport, exercise and health: From process to product. London: Routledge.

Tweedy, S. et al. (20I4): "Paralympic classification: conceptual basis, current methods, and research update". Paralympic sports medicine and science, 6 (8): I I-I7.

Tweedy, S. y Vanlandewijck, Y. (2OII): "International Paralympic committee position stand- background and scientific principles of classification in Paralympic sport". British journal of sports medicine, 45: 259-269.

Tweedy, S. M. (2002): “Taxonomic theory and the ICF: foundations for a unified disability athletics classification". Adapted physical activity quarterly, I9 (2): 220-237. 